\title{
Can Galactic Observations Be Explained by a Relativistic Gravity Theory?
}

\author{
Vadim V. Zhytnikov* and James M. Nester \\ Department of Physics, National Central University, Chung-Li, Taiwan 32054
}

(June 1994)

\begin{abstract}
We consider the possibility of an alternative gravity theory explaining the dynamics of galactic systems without dark matter. From very general assumptions about the structure of a relativistic gravity theory we derive a general expression for the metric to order $(v / c)^{2}$. This allows us to compare the predictions of the theory with various experimental data: the Newtonian limit, light deflection and retardation, rotation of galaxies and gravitational lensing. Our general conclusion is that the possibility for any gravity theory to explain the behaviour of galaxies without dark matter is rather improbable. PACS numbers: 04.50.+h, 04.25.Nx, 95.35.+d
\end{abstract}


Einstein's gravity theory and some other alternative gravity models are in good agreement with the experimental data in the solar system and the laboratory [1]. However, the behavior of galactic systems poses a great challenge to gravity theories. For virtually all spiral galaxies the tangential rotational velocity curves tend toward some constant value. This fact is in sharp contradiction with the visible star (luminosity) distribution and the laws of Newtonian dynamics. The stars in the outer parts of galaxies rotate several times faster than predicted by the standard gravity theory. A similar problem is observed in gravitational lensing [2]. Just as in the solar system problems of the past century (concerning the orbits of Uranus and Mercury), there are two ways to resolve these difficulties.

One, the most widely adopted, is the dark matter hypothesis [3]. It is presumed that the visible stars are imbedded in a massive nearly spherical halo of nonluminous matter. The mass of the halo varies from one galaxy to another but generally it constitutes about $90 \%$ of the total mass. This hypothesis explains the flat rotational curves of galaxies. Yet it has its own troubles, in particular (i) no good model for the formation of the dark halo is known, and (ii) after much effort and many proposals no known form of matter has yet given a satisfactory model for the massive halo. (The few recently observed cases of gravitational microlensing [4] are as yet far from conclusive evidence for the dark matter explanation).

The second way is to assume that for galactic distances Newton's gravity law is no longer valid. This possibility has also been the subject of some discussions [5 \&]. In particular it was shown [9] that a modified gravitational potential of the form

$$
\varphi=\frac{-G M}{r(1+\alpha)}\left[1+\alpha e^{-r / r_{0}}\right]
$$

where $\alpha=-0.9, r_{0} \approx 30 \mathrm{kpc}$ can explain flat rotational curves for most of the galaxies. The potential (11) differs from the usual one by an extra exponential term. For the solar system this term equals 1 with high accuracy and (11) reduces to the standard form $\varphi=$ $-G M / r$ but for distances significantly greater than $30 \mathrm{kpc}$ the exponential term vanishes and we have once again a Newtonian potential $\varphi=-G M /(1+\alpha) r$ but now with an approximately ten times bigger gravitational constant $G /(1+\alpha)$. Besides (11) several other 
modified gravitational models were considered in the literature [5]. Most were introduced purely phenomenologically without derivation from some gravity theory. All of these models and also various attempts to construct nonrelativistic gravity theories [5] have the same trouble. They cannot describe the motion of light without additional assumptions. A description of light requires a relativistic gravity model.

We investigate the general possibility of constructing a relativistic gravity theory which can explain galactic mysteries and other experimental data like the classical solar system tests [1]. We formulate some very general postulates about the structure of the theory:

(i) Gravitational phenomena are described by the metric of space-time $g_{\mu \nu}$ and possibly some other set of fields $\Psi_{A}$. The theory is invariant under general coordinate transformations.

(ii) The trajectories of (structureless) massive test particles and light are timelike and null geodesics of the metric $g_{\mu \nu}$ respectively.

(iii) The source for the gravitational fields $g_{\mu \nu}$ and $\Psi_{A}$ are the energy-momentum tensor $T_{\mu \nu}$ and some current $J_{A}$. For the solar system and galaxies these sources can be taken in the form $T_{\mu \nu}=T_{00}=\rho, J_{A}=0$ in the $(v / c)^{2}$ approximation.

(iv) The theory has a good linear approximation.

(v) Flat spacetime $g_{\mu \nu}=\eta_{\mu \nu}=\operatorname{diag}(-1,1,1,1)$ and $\Psi_{A}=\Psi_{A}^{0}\left(\Psi_{A}^{0}\right.$ some constant or almost constant field) can be considered as the background field configuration for the solar system and galactic scales.

(vi) The theory does not possess any unusual gauge freedom for the metric field besides general coordinate invariance. Any gauge freedom for the field $\Psi_{A}$ is fixed by an appropriate gauge fixing condition.

(vii) The theory is not a higher-derivative theory.

Let us briefly discuss postulates (i)-(vii). The postulates (i),(ii) are the usual postulates of the so called metric theory of gravity [1]. Postulate (iii), especially the condition $J_{A}=0$, is of crucial importance for our study. First, it allows us to make definite predictions about the post-Newtonian approximation of the theory without needing detailed information about 
its structure. Second, as we'll see below, it ensures that the theory does not violate the equivalence principle. The nature of the current $J_{A}$ may be different; for some models it may be absent explicitly. In particular, for the Brans-Dicke [1] theory $\Psi_{A}$ is the scalar field and it does not have any corresponding matter source. However, for the Poincaré gauge theory of gravitation [10] $\Psi_{A}$ is the space-time torsion and the current is the spintensor of matter which vanishes to a high approximation since both the solar system and the galaxies do not contain large amounts of spin-polarized matter. Postulate (iv) excludes from our considerations all essentially nonlinear theories for which the linear approximation is invalid. Postulate (v) means that we neglect global cosmological effects. Postulate (vi) ensures that gravitational equations are nondegenerate. The assumption about the absence of extra gauge freedom for the metric is quite natural since such invariance normally imposes unphysical constraints on the energy-momentum tensor of matter fields. Postulate (vii) excludes from our consideration theories with Green functions of the form $1 /\left(\square-m^{2}\right)^{n}, n \geq 2$. These postulates are actually not too restrictive. For example they allow a large class of geometric gravity theories derived from Lagrangians depending on the metric and the connection through the torsion, curvature and non-metricity.

We are going to compare the predictions of any gravity theory which satisfies postulates (i)-(vii) with the experimental data from the solar system and the galaxies. A key point is that all of these systems are essentially post-Newtonian slow-motion, weak-gravitationalfield systems [1]. Hence we can consider our theory in the linearized approximation and we can use the small post-Newtonian parameter $\varphi \approx v^{2} \ll 1$ (we assume $c=\hbar=1$ ) in order to solve the gravitational equations approximately $(\varphi$ is a typical gravitational potential and $v$ a typical velocity in the system). Observe, that $G T_{00}=G \rho=O\left(v^{2}\right)[1]$ and, therefore, the leading corrections for the $g_{\mu \nu}, \Psi_{A}$ are $O\left(v^{2}\right)$. Thus, we can represent our fields in the form

$$
g_{\mu \nu}=\eta_{\mu \nu}+h_{\mu \nu}, \quad \Psi_{A}=\Psi_{A}^{0}+\psi_{A}
$$

where $h_{\mu \nu}, \psi_{A}=O\left(v^{2}\right)$. We are interested in computing only the metric since the test bodies are not sensitive to the other fields. It is well known [1] that the first post-Newtonian 
correction for the equations of motion of massive test particles depends on $h_{00}$ only. This is just the Newtonian approximation and $-\frac{1}{2} h_{00}$ is the gravitational potential. But the postNewtonian equations for light include both the $h_{00}$ and $h_{i k}, i, k=1,2,3$ components of the metric (the gravitational potential alone cannot describe the motion of light!).

The $h_{\mu \nu}, \psi_{A}$ can be obtained from the linearized equations of the theory. The invertible linear operator $\mathcal{D}$ of these equations is constructed with the help of $\eta_{\mu \nu}, \partial_{\mu}$ and $\Psi_{A}^{0}$. At this stage we work with the weak field relativistic approximation. In the end we obtain the required post-Newtonian approximation by dropping all terms with time derivatives $\partial_{0}$ and replacing $\square$ by $\Delta$. We assume that $\mathcal{D}$ does not contain terms of the form $\Psi_{\alpha}^{0} \partial^{\alpha}$. Such terms usually violate spatial isotropy, verified experimentally with rather high accuracy [1]. Now we can use the spin projection operators [11] and decompose our equations on the independent spin sectors. In general the field $h_{\mu \nu}$ can contain contributions of 4 different kinds of particles of spin $2^{+}, 1^{-}, 0^{+}, 0^{+}$. The corresponding projectors read

$$
\begin{aligned}
& P^{2^{+}}=\theta_{\mu}^{\alpha} \theta_{\nu}^{\beta}-\frac{1}{3} \theta_{\mu \nu} \theta^{\alpha \beta}, P^{1^{-}}=2 \theta_{(\mu}^{\alpha} \omega_{\nu)}^{\beta}, \\
& P_{1}^{0^{+}}=\frac{1}{3} \theta_{\mu \nu} \theta^{\alpha \beta}, P_{2}^{0^{+}}=\omega_{\mu \nu} \omega^{\alpha \beta}, \\
& P_{12}^{0^{+}}=\theta_{\mu \nu} \omega^{\alpha \beta} / \sqrt{3}, P_{21}^{0^{+}}=\omega_{\mu \nu} \theta^{\alpha \beta} / \sqrt{3},
\end{aligned}
$$

here $\omega_{\mu \nu}=\partial_{\mu} \partial_{\nu} / \square$, and $\theta_{\mu \nu}=\eta_{\mu \nu}-\omega_{\mu \nu}$. The subscripts 1,2 in the spin $0^{+}$sector label two different kinds of particles of this type. A similar spin decomposition exists for the field $\psi_{A}$ but we are not interested in its detailed contents since the corresponding source $J_{A}$ vanishes. Nonzero contribution to the metric for the source $T_{00}=\rho$ can come only from $P^{2^{+}} T_{\mu \nu}$ and $P_{1}^{0^{+}} T_{\mu \nu}$. All other projectors produce terms which either vanish in the given approximation or can be eliminated by an appropriate choice of coordinate system. In general each spin sector can contain several different particles. The form of the linearized equations in the spin $2^{+}$sector is

$$
\left(\begin{array}{ccc}
M_{00} P_{0} & M_{01} P_{01} & \cdots \\
M_{10} P_{10} & M_{11} P_{1} & \cdots \\
\ldots & \ldots & \cdots
\end{array}\right)\left(\begin{array}{c}
P_{0} h_{\mu \nu} \\
P_{1} \psi_{A} \\
\vdots
\end{array}\right)=\left(\begin{array}{c}
P_{0} T_{\mu \nu} \\
0 \\
\vdots
\end{array}\right)
$$


where the coefficients $M_{i k}$ are scalar polynomial functions of the operator $\square=\partial_{\alpha} \partial^{\alpha}$ (here for simplicity we omit the superscript $2^{+}$and denote $P^{2^{+}} \equiv P_{0}$, the subscripts $1,2, \ldots$ label different $2^{+}$modes $)$. The determinant of the matrix $M_{i j}$ has the form $\prod\left(\square-m_{p}^{2}\right)^{q}$. The constants $m_{p}$ play the role of "masses" for the propagating linearized modes of our alternative gravity theory. Due to the orthogonality and completeness properties of the spin projectors the solution of the equations (3) can be obtained merely by calculating the inverse matrix $N_{i k}=M_{i k}^{-1}$. We are interested to know $N_{00}$ only; its general form is $N_{00}=\sum \sigma_{p} /\left(\square-m_{p}^{2}\right)$. Finally we have to replace all operators $1 /\left(\square-m^{2}\right)$ by $1 /\left(\Delta-m^{2}\right)$ which leads to Yukawa exponential potentials. The analysis of the spin $0^{+}$sector is completely analogous. Hence, we obtain a general form for the metric

$$
\begin{aligned}
& g_{00}=-1+2\left[\left(\sigma_{0}+\tau_{0}\right) U+\sum_{p=1}^{n_{2}} \sigma_{p} U_{p}+\sum_{q=n_{2}+1}^{n_{2}+n_{0}} \tau_{q} U_{q}\right] \\
& g_{i k}=\delta_{i k}\left(1+2\left[\left(\frac{\sigma_{0}}{2}-\tau_{0}\right) U+\sum_{p=1}^{n_{2}} \frac{\sigma_{p}}{2} U_{p}-\sum_{q=n_{2}+1}^{n_{2}+n_{0}} \tau_{q} U_{q}\right]\right) .
\end{aligned}
$$

Here the constants $\sigma_{p}, \tau_{p}$ depend on the parameters of the concrete model and/or the constant field $\Psi_{A}^{0}$. The constants $\sigma_{p}$ and $\tau_{p}$ represent the contributions of the spin $2^{+}$and spin $0^{+}$modes respectively. The Newtonian potential $U$ and exponential potentials $U_{p}$

$$
U=G \int \frac{\rho^{\prime}}{\left|\vec{x}-\vec{x}^{\prime}\right|} d^{3} x^{\prime}, \quad U_{p}=G \int \frac{\rho^{\prime} \mathrm{e}^{-m_{p}\left|\vec{x}-\vec{x}^{\prime}\right|}}{\left|\vec{x}-\vec{x}^{\prime}\right|} d^{3} x^{\prime},
$$

correspond to the massless and massive modes with mass $m_{p}$. Now all information about a particular gravity theory is packed into several constants $\tau_{i}, \sigma_{k}, m_{p}$.

We can compare the metric (雨) with the standard metric of the parametrized postNewtonian (PPN) formalism in the same $O\left(v^{2}\right)$ approximation [1]:

$$
g_{00}=-1+2 U, \quad g_{i k}=\delta_{i k}(1+2 \gamma U) .
$$

Here the coefficient of $U$ in $g_{00}$ is fixed by the Newtonian limit and the experimental value for $\gamma=1 \pm 10^{-3}$ comes from light deflection and retardation experiments in the solar system [1]. The only essential difference between (4) and (囵) is that the standard PPN formalism 
does not take into account a possible contribution from massive modes. Of course, the influence of massive exponential potentials on the predictions of the gravity model depends on the concrete values of the masses $m_{p}$. If the mass of the mode is large enough, i.e., such that $1 / m_{p}$ is significantly less than $1 \mathrm{~cm}$, then the contribution of $U_{p}$ can not be observed in gravity experiments and our metric reduces effectively to (6). On the other hand if $1 / m_{p}$ is larger than the typical size of a galaxy then $U_{p} \approx U$ for galactic and shorter distances and we are left once again with the metric (6). In principle $1 / m_{p}$ could be about the size of the Earth or the solar system but in this case experimental data impose very strong restrictions on the magnitude of the constants $\sigma_{p}, \tau_{p}$ [12]. For example if $1 / m_{p} \approx 10^{13} \mathrm{~cm}$ then $\sigma_{p}, \tau_{p}<10^{-8}$.

Therefore, we have hopes to explain the dynamics of galaxies if $1 / m_{p}$ has an intermediate value significantly larger than the size of the solar system but not greater than the typical size of a galaxy (compare with (1) ). For our purposes it is sufficient to consider the simple case with two massive particles of spins $2^{+}$and $0^{+}$with approximately equal masses $m_{1} \approx m_{2} \approx$ $10^{-26} \mathrm{eV}$. Thus, we have (仼) with four unknown constants $\sigma_{0}, \tau_{0}, \sigma_{1}, \tau_{2}$. The experimental data imposes constraints on these parameters. In the solar system $U_{1} \approx U_{2} \approx U$ and we have

$$
\sigma_{0}+\tau_{0}+\sigma_{1}+\tau_{2}=1, \quad \frac{1}{2}\left(\sigma_{0}+\sigma_{1}\right)-\left(\tau_{0}+\tau_{2}\right)=1
$$

where the first condition ensures the correct Newtonian limit while the second follows from experiments with light. Now let us consider distances larger than 30kpc. For these distances $U_{1} \approx U_{2} \approx 0$. As was mentioned above in order to reproduce the flat rotational curves of galaxies without dark matter as far as is known we only need the gravitational interaction to be approximately 10 times stronger for large distances

$$
\sigma_{0}+\tau_{0} \approx 10, \quad \frac{1}{2} \sigma_{0}-\tau_{0} \approx 10
$$

Here the second conditions follow from gravitational lensing, since it is known [2] that the observed lensing is in conformity with the predictions of Einstein's gravity with dark matter. 
Thus, we have once again an approximately ten times stronger effective coupling constant for light. Solving (7), (8) one has

$$
\sigma_{0} \approx \frac{40}{3}, \quad \tau_{0} \approx-\frac{10}{3}, \quad \sigma_{1} \approx-12, \quad \tau_{2} \approx 3
$$

Any gravity model with the parameters (9) should be in good correspondence with experimental data in the solar system and should explain the behaviour of stars and light in galaxies with reasonable accuracy. Probably if we include more spin $0^{+}$and $2^{+}$particles of various masses we could fit more details of the galactic rotation curves [8]. This hypothetical model is not so simple: the gravity theory should include, besides the usual graviton, at least two extra massive very light particles [13]. The most serious trouble with such a theory comes from the negative sign of $\tau_{0}$ and $\sigma_{1}$. In accordance with a general theorem 14] the sign of these constants must be positive for even spin fields and negative for odd spin fields. A wrong sign results in a propagating mode carrying negative energy; this is considered unacceptable in a theory. There is still a small possibility of escaping this problem. It has been suggested that if the theory contains several particles with the same spin then the conditions on the parameters might be weakened [8]. In our opinion the possibility of successfully matching the galactic rotation curves while avoiding negative energy modes seems remote.

On the other hand, a negative sign for the coupling constant is natural for odd spin particles; initially it was suggested that the exponential term in (11) is mediated by a spin 1 vector particle [9]. In our scheme one can reproduce such a contribution if and only if $J_{A} \neq 0$. In particular, it was suggested that $J_{A}$ may be proportional to baryonic charge [15]. However, the baryonic charge to mass ratio varies from one body to another. Therefore, such an interaction is no longer universal and violates the weak equivalence principle [16] which has been verified experimentally to a very high accuracy [1]. Now the importance and fundamental nature of postulate (iii) becomes clear. It ensures the universality of the gravitational interaction. All models which satisfy this condition should not have trouble with the equivalence principle at least on the modern experimental level. 
We have compared our model only with part of the available experimental data. Even if one overlooks the problem with the wrong sign of the coupling constants the model must describe correctly, in addition to the already considered effects, the perihelion shift of Mercury, the energy loss of the binary-pulsar, and cosmological observations. Comparison with these data cannot be performed with the help of linearized equations and requires a more detailed consideration for each particular gravity theory. Of course, such comparison probably result in additional perhaps severe restrictions on the theory under consideration (see e.g. [17] which discuss cosmological restrictions).

Although our scheme covers a large class of the theories yet we can not exclude the possibility of constructing a model with the desired properties which violates one of our postulates. We want to mention briefly some possibilities which have been proposed.

1. It has been shown 18 that a cosmological constant $\Lambda \approx 10^{-52} \mathrm{~cm}^{-2}$ is able to explain the flat rotational curves of galaxies. This value of $\Lambda$ is ten times bigger than the cosmologically acceptable limit.

2. Consideration of quantum corrections to the Newtonian potential may result in some additional logarithmic long range terms [19]. The applicability of these results to galactic distances is not obvious.

3. A quadratic in curvature lagrangian can also produce modified gravitational potentials with extra long range terms [7]. This model has higher derivatives equations and requires a traceless energy-momentum tensor.

4. It is possible to construct an essentially nonlinear theory with a non-quadratic kinetic term in the Lagrangian. This model explains the dynamics of galaxies due to the nonlinear nature of the equations in the regime of small accelerations [16,5]. It was observed that such a theory may have troubles with faster-than-light waves.

Although all these models explain the rotation of galaxies they have their own weak points, moreover they must be capable of predicting correctly other gravitational effects. Therefore we conclude that the possibility of explaining galactic mysteries with the help of a modified gravity theory looks quite improbable. 
This work has been supported by the National Science Council of the Republic of China under contracts No NSC 83-0208-M008-014 and NSC 83-0208-M008-028. We thank the referee for informing us of Ref. [17]. 


\section{REFERENCES}

* Permanent address: Nuclear Safety Institute, Bolshaya Tulskaya 52, Moscow 113191, Russia.

[1] C. M. Will, Theory and experiment in gravitational physics (Cambridge Univ. Press, Cambridge, 1981, 1993).

[2] A. Dar, Nuclear Phys. B (Proc. Suppl.), 28A, 321 (1992).

[3] K. M. Ashman, Publications of the Astron. Soc. of the Pacific. 104, 1109 (1992).

[4] C. Alcock at al., Nature, 365, 621 (1993); E. Aubourg at al., Nature, 365, 623 (1993).

[5] J. D. Bekenstein, in Proc. 2nd Canadian Conf. on General Relativity and Relativistic Astrophysics, ed. C. Dyer and T. Tupper (Singapore, World Scientific, 1988) p. 68.

[6] R. Hammond, Dark Matter or New Gravity? in Matters of gravity electronic newsletter, ed. J. Pullin, vol. 3 (1994) p. 25.

[7] P. D. Mannheim, Found. Phys., 24, 487 (1994); P. D. Mannheim and D. Kazanas, Gen. Relativ. Gravit., 26, 337 (1994).

[8] D. H. Eckhardt, Phys. Rev. D48, 3762 (1993).

[9] R. H. Sanders, Astron. Astrophys. 136, L21 (1984); 154, 135 (1986).

[10] F. W. Hehl, P. von der Heyde, G. D. Kerlik, and J. M. Nester, Rev. Mod. Phys. 48, $393(1976)$.

[11] R. J. Rivers, Nuovo Cimento, 34, 386 (1964); P. van Nieuwenhuizen, Nucl. Phys. B60, $478(1973)$.

[12] E. Fischbach and C. Talmadge, in Proc. of Sixth Marcel Grossmann Meeting, eds. H. Sato and T. Nakamura (world Scientific, Singapore, 1992) p. 1122.

[13] A concrete example is the Poincaré gauge theory which has up to 3 extra particles. See 
M. S. Gladchenko and V. V. Zhytnikov, Phys. Rev. D, October 1994.

[14] K. Jagannathan and L. P. S. Singh, Phys. Rev. D33, 2475 (1986).

[15] J. Scherk, Phys. Lett. 88B, 265 (1979).

[16] R. H. Sanders, Mon. Not. R. Astr. Soc. 223, 539 (1986).

[17] T. Jolicoeur, in New and Exotic Phenomena. Proc. of the 7th Moriond Workshop, eds. O. Fackler and J. Tran Thanh Van (Editions Frontiers, 1987) p. 203.

[18] E. Gessner, Astrophys. and Space Sci. 194, 197 (1992).

[19] D. A. R. Dalvit and F. D. Mazzitelli, Running Coupling Constants, Newtonian Potential and non localities in the Effective Action (unpublished). 